\title{
Depressão no climatério: indicadores biopsicossociais
}

\author{
Depression during the climacteric: biopsychosocial indicators
}

\author{
Andréia Fernanda Nievas, Antonia Regina Ferreira Furegato, Odilon lannetta \\ e Jair Licio Ferreira Santos
}

\begin{abstract}
Resumo
Objetivo: Identificar a presença de sintomas depressivos em mulheres no climatério, analisando os indicadores biopsicossociais relacionados. Métodos: Aplicaram-se o Questionário de Identificação e Contexto da Mulher no Climatério (ICMC) e o inventário de Beck a 30 mulheres em primeiro atendimento no Ambulatório Multidisciplinar do Climatério do Hospital das Clínicas da Faculdade de Medicina de Ribeirão Preto da Universidade de São Paulo (HCRP/FMRP/USP). Os dados foram estatisticamente trabalhados por análise bivariada e multivariada. Resultados: Observa-se que, de acordo com o inventário de Beck, foi identificada depressão em 14 das 30 mulheres atendidas no ambulatório no período de novembro de 2003 a dezembro de 2004. Conclusão: Os resultados mostraram que os sintomas depressivos estavam aumentados em mulheres na faixa dos 40 aos 49 anos, não-brancas, que tinham companheiro pertencente ao grupo biológico $\mathrm{B}$ ou $\mathrm{C}$, que tinham problemas com o cônjuge, que vivenciaram hábito de beber, situação de óbito recentemente e/ou desemprego na família.

Palavras-chave: depressão, climatério, enfermagem.
\end{abstract}

\begin{abstract}
Objective: This study aimed to identify the presence of depressive symptoms in women during the climacteric and to analyze the related biopsychosocial indicators. Methods: The Questionnaire for the Identification and Context of Women during the Climacteric and Beck's Inventory were applied to 30 women who first attended the Multidisciplinary Menopause Outpatient Clinic at the HCRP/FMRP/ USP, Brazil. Data were statistically processed through bivariate and multivariate analysis. Results: According to the Beck Inventory the presence of depression was identified in 14 of the 30 women attended in that outpatient clinic from November 2003 to December 2004. Conclusion: Results showed the increased presence of depressive symptoms in non-white women between 40 and 49 years old, who had a partner, belonged to biological groups $\mathrm{B}$ or $\mathrm{C}$, were going through problems with their partner, had drinking habits and had faced a situation of recent death or unemployment in the family.
\end{abstract}

Key words: depression, climacteric, nursing.

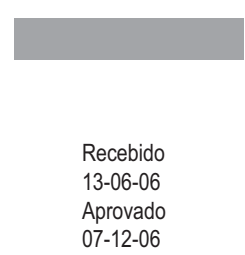

Escola de Enfermagem de Ribeirão Preto da Universidade de São Paulo (EERP/USP) (Nievas AF, Furegato ARF) Faculdade de Medicina de Ribeirão Preto da Universidade de São Paulo (FMRP/USP) (lannetta O, Santos ILF) Estudo apoiado pela Fundação Coordenação de Aperfeiçoamento de Pessoal de Nível Superior (CAPES).

Correspondência para: Antonia Regina Ferreira Furegato

Escola de Enfermagem de Ribeirão Preto da Universidade de São Paulo

Avenida Bandeirantes, 3.900 - Campus - 14040-902 - Ribeirão Preto-SP

e-mail: furegato@eerp.usp.br 


\section{Introdução}

O estudo da depressão na fase do climatério é antigo. Foi descrito na Inglaterra, no final do século XIX, como uma forma de melancolia. $O$ conceito de melancolia involutiva foi introduzido há 80 anos por Kraepelin como uma entidade nosológica distinta, diferenciando-a da psicose maníaco-depressiva por critérios evolutivos. Esse conceito foi muito questionado e Kraepelin o reformulou, concluindo que o surgimento desse quadro clínico após os 45 anos era devido ao aumento do número de casos de doença afetiva, e não a uma nova condição clínica. Vários autores continuam refutando e outros defendendo esse conceito. Através dos vários estudos realizados sobre os sintomas depressivos não se encontrou base científica para apoiar um conceito único (Appolinário, 1999).

O climatério é a fase da existência da mulher compreendida entre o final da vida reprodutora e a senescência, marcada por alterações importantes como a menopausa (Beck et al., 1961; Biffi, 1991; Carranza-Lira et al., 1993; Dennersttein et al., 1999).

O climatério, como uma fase da vida da mulher na qual ocorrem principalmente mudanças hormonais com conseqüências físicas, psicológicas e sociais, é um momento de considerável estresse e, portanto, de risco para o desenvolvimento da depressão. Muitos estudos têm sido feitos nessa área (Carranza-Lira e Valentino-Figuero, 1999; Dias et al., 2002; Freeman et al., 2004; Genazzani et al., 2002; Gorenstein e Andrade, 1998; Halbe, 2000; lannetta et al., 1997).

Ao lado das alterações biológicas, outros fatores devem ser considerados na interação entre depressão e climatério, como status ocupacional, estado civil, ocorrência de eventos importantes na vida e episódio anterior de depressão (Gorenstein e Andrade, 1998; Lamberts et al., 1997).

Como a expectativa de vida vem aumentando expressivamente, é possível admitir que as mulheres viverão um terço de suas vidas após a menopausa (Maartens et al., 2002). Portanto a saúde e a qualidade de vida das mulheres merecem atenção especial para que, nessa fase, possam viver de maneira tão saudável quanto em outras fases de sua vida. Os objetivos deste estudo foram: a) caracterizar a população de mulheres no climatério quanto a idade, estado civil e nível de escolaridade; b) identificar a presença de sintomatologia sugestiva de depressão entre mulheres no climatério e indicadores biopsicossocias relacionados.

\section{Métodos}

\section{Pressupostos do estudo}

A mulher no climatério apresenta com freqüência sintomatologia sugestiva de depressão. Além das questões biológicas que podem interferir nesse fenômeno, a rede de relações e atividades psicossociais pode alterar a presença de sintomas depressivos.

\section{Local}

A pesquisa foi realizada no Ambulatório Multidisciplinar do Climatério (ACLI) do Hospital das Clínicas da Faculdade de Medicina de Ribeirão Preto (HC/FMRP).

\section{População}

A população estudada foi composta por mulheres atravessando o período de climatério. Foram entrevistadas 30 mulheres em primeiro atendimento no ACLI, as quais concordaram em participar da pesquisa no período de novembro de 2003 a dezembro de 2004.

\section{Instrumentos}

Foram utilizados dois instrumentos para essa pesquisa: 1. o Questionário de Identificação e Contexto da Mulher no Climatério (ICMC), que consta de identificação do sujeito (dados biopsicológicos), informações clínicas e contextualização (questões socioeconômicas e culturais);

2. o inventário de Beck (Miranda e Figueira, 1999), uma escala psicométrica composta de 21 itens que se referem à sintomatologia depressiva: tristeza, pessimismo, sensação de fracasso, falta de satisfação, sensação de culpa, sensação de punição, autodepreciação, auto-acusações, idéias suicidas, crises de choro, irritabilidade, retração social, indecisão, distorção da imagem corporal, inibição para o trabalho, distúrbio do sono, fadiga, perdas de apetite e de peso, preocupação somática e diminuição da libido.

O inventário de Beck foi traduzido e validado para o português por Gorenstein e Andrade (Nicol-Smith, 1996) numa população de estudantes universitários e vem sendo amplamente aplicado em muitas pesquisas, inclusive na enfermagem (Oliveira e Lemgruba, 2001; Perez et al., 1997; Richardson e Robinson RD, 2000.

\section{Procedimentos na coleta dos dados}

A coleta de dados ocorreu de novembro de 2003 a dezembro de 2004 na primeira consulta de cada mulher agendada no ACLI. Foram explicados os objetivos da pesquisa, o objeto de estudo, a forma de participação no estudo e o uso das informações. Após os esclarecimentos, os questionários eram lidos e as respostas, assinaladas.

\section{Aspectos éticos}

O projeto desta pesquisa passou por análise e aprovação do Comitê de Ética do Hospital das Clínicas da FMRP/USP. Todas as mulheres foram devidamente esclarecidas e sua autorização individual foi assinada no termo de consentimento livre e esclarecido.

\section{Procedimentos da análise dos dados}

Os resultados foram analisados estatisticamente através de análises bivariada e multivariada, comparando os sintomas indicativos de depressão com as variáveis idade, estado civil, grau de instrução, terapia de reposição hormonal (TRH), grupos biológicos e demais indicadores psicossociais.

\section{Resultados}

\section{Dados dos perfis sociodemográfico e clínico}

A idade das 30 mulheres entrevistadas variou de 40 a 70 anos, sendo que a maior porcentagem $(46,7 \%)$ encontrava-se na faixa entre 40 e 50 anos. 
Esse grupo de mulheres foi composto por maioria branca (86\%), sendo que $63,3 \%$ delas moram com companheiro. Desse total, uma é analfabeta, dez completaram o curso primário, cinco a 8a série, nove têm o ensino médio completo e cinco possuem ensino superior. Em relação à situação de trabalho, observa-se que quatro não trabalham, 16 exercem atividade remunerada, três têm trabalho eventual e sete são aposentadas.

Em outro momento da entrevista, quando indagadas sobre ocupação, 19 mulheres disseram não tê-la de nenhum tipo. Entre aquelas que relataram ocupação, quatro cuidavam da casa, uma fazia artesanato e outras duas crochê, uma era auxiliar de cabeleireiro, uma era manicure, uma relatou que fazia tricô, crochê e costura e uma delas vendia roupas intimas.

As participantes foram classificadas em três grupos biológicos de acordo com o período do climatério ( $A, B$ e $C)$, conforme classificação utilizada no ACLI:

- grupo A: ciclos menstruais regulares e com sintomas de climatério;

- grupo B: ciclos menstruais irregulares e presença de sintomas da menopausa;

- grupo C:

- C 1: amenorréia de 12 a 23 meses;

- C 2: amenorréia de 2 anos até 2 anos e 11 meses;

- C 3: amenorréia de 3 anos até 3 anos e 11 meses;

- C 4: amenorréia de 4 anos até 4 anos e 11 meses;

- C 5: amenorréia de 5 anos ou mais.

Das 30 mulheres entrevistadas, sete faziam reposição hormonal, seis usavam corticosteróide e duas, antidepressivo. Somente oito não utilizavam regularmente nenhum tipo de medicação no momento da entrevista. Observa-se na Tabela 1 que mais de $60 \%$ enquadravamse no grupo $C$.

Duas pessoas da população estudada tinham diagnóstico de depressão no prontuário médico. Entretanto, na ficha do ACLI, entre as queixas emocionais, 33,3\% relataram que se sentiam deprimidas.

\section{Sinais indicativos de depressão entre as mulheres climatéricas}

Para uma população diagnosticada ou que procura serviços especializados, os pontos de corte recomendados para se identificar a depressão são diferentes dos da população em geral, não diagnosticada (Nicol-Smith, 1996). Esse é o caso das mulheres em atendimento no ambulatório, cujo ponto de corte recomendado é de até 15 pontos sem depressão; de 16 a 20, disforia ou depressão leve; de 21 a 29, depressão moderada; e acima de 30 , depressão grave.

$\mathrm{Na}$ Tabela 2 observa-se o resultado do teste (inventário de Beck) que identificou a presença de depressão em 14 das 30 mulheres atendidas no ambulatório no período de novembro de 2003 a dezembro de 2004.

Ao comparar (por meio de análises bivariada e multivariada) a presença ou ausência de sintomas sugestivos de depressão com as características de identificação e os dados clínicos e psicossociais das mulheres desse estudo, observa-
Tabela 1. Distribuição das freqüências das mulheres no climatério segundo seus respectivos grupos biológicos

\begin{tabular}{|c|c|c|}
\hline Grupo biológico & Freqüência & Porcentagem \\
\hline A & 6 & 20 \\
\hline B & 5 & 16,7 \\
\hline $\mathrm{C}: \mathrm{C1}$ & 1 & 3,3 \\
\hline $\mathrm{C} 2$ & 4 & 13,4 \\
\hline C3 & 1 & 3,3 \\
\hline C4 & 1 & 3,3 \\
\hline C5 & 12 & 40 \\
\hline Total & 30 & 100 \\
\hline
\end{tabular}

se que a presença de disforia/depressão nas mulheres com mais de 40 anos pode estar associada ao fato de que, nesse período, a mulher estaria mais próxima do evento menopausa, responsável por irregularidades menstruais devido às alterações hormonais. A presença de sintomas nessa fase condiz com os achados da categoria do grupo biológico $B$, no qual $80 \%$ das mulheres apresentam disforia ou sinais sugestivos de depressão (Tabela 3).

Na Tabela 4 estão registrados os resultados da regressão logística por passos. A variável cor foi retirada por problemas de estimação não especificados. As variáveis: problemas com cônjuge, desemprego, UBS (Unidades Básicas de Saúde) e PSF (Programa Saúde da Família) apresentaram observações insuficientes para os cruzamentos múltiplos, tendo sido eliminadas no processo. A variável ocorrência de

Tabela 2. Distribuição da freqüência de depressão nas mulheres na fase do climatério atendidas no ACLI/HCRP/USP

\begin{tabular}{lcc}
\hline \multicolumn{1}{c}{ Depressão } & Freqüência & Porcentagem \\
\hline Sem depressão & 16 & 53,3 \\
Disforia/depressão leve & 7 & 23,3 \\
Depressão moderada & 6 & 20 \\
Depressão grave & 1 & 3,3 \\
\hline Total & 30 & 99 \\
\hline
\end{tabular}

óbito não satisfez o critério de permanência e foi desprezada. As demais variáveis independentes satisfizeram o critério de manutenção e entraram para o modelo final, conforme se observa na Tabela 4.

Pelos resultados, a menor significância observada foi de $15,6 \%$ (grupo etário) e a maior, de 6,2\% (grupos biológicos). A maior razão de risco é a da variável hábito de beber. Conviver com o hábito apresentou, na amostra, 16,88 vezes mais chances de ter depressão. Ter companheiro aumenta a chance em 7,96; ser jovem aumenta em 6,92; e ser do grupo biológico $B$ ou $C$ aumenta em 5,65. 
Tabela 3. Freqüência e porcentagem da presença de depressão segundo variáveis independentes e significâncias do teste de Fisher

\begin{tabular}{|c|c|c|c|c|}
\hline \multirow{2}{*}{ Categoria } & \multicolumn{2}{|c|}{ Disforia/depressão } & \multirow{2}{*}{ Total } & \multirow{2}{*}{$\begin{array}{l}\text { Teste de } \\
\text { Fisher }\end{array}$} \\
\hline & Freqüência & Porcentagem & & \\
\hline \multicolumn{5}{|l|}{ Grupo etário } \\
\hline 40 a 49 anos & 8 & 66,67 & 12 & 0,078 \\
\hline 50 ou mais & 6 & 33,33 & 18 & 0,078 \\
\hline \multicolumn{5}{|l|}{ Companheiro } \\
\hline Não & 3 & 27,27 & 11 & 0,107 \\
\hline Sim & 11 & 57,89 & 19 & 0,107 \\
\hline \multicolumn{5}{|l|}{ Cor } \\
\hline Branca & 10 & 38,46 & 26 & 0,037 \\
\hline Não-branca & 4 & 100 & 4 & 0,037 \\
\hline \multicolumn{5}{|c|}{ Hábito de beber } \\
\hline Não & 11 & 42,31 & 26 & 0,249 \\
\hline Sim & 3 & 75 & 4 & 0,249 \\
\hline \multicolumn{5}{|c|}{ Ocorrência de óbito } \\
\hline Sim & 5 & 71,43 & 7 & 0,143 \\
\hline Não & 9 & 39,13 & 23 & 0,143 \\
\hline \multicolumn{5}{|c|}{ Grupo biológico } \\
\hline A & 1 & 16,67 & 6 & 0,135 \\
\hline B & 4 & 80 & 5 & 0,135 \\
\hline C & 9 & 47,37 & 19 & 0,135 \\
\hline \multicolumn{5}{|l|}{ UBS } \\
\hline Sim & 10 & 55,56 & 18 & 0,206 \\
\hline Não & 4 & 33,33 & 12 & 0,206 \\
\hline \multicolumn{5}{|l|}{ PSF } \\
\hline Sim & 1 & 20 & 5 & 0,209 \\
\hline Não & 13 & 52 & 25 & 0,209 \\
\hline \multicolumn{5}{|l|}{ Desemprego } \\
\hline Sim & 3 & 100 & 3 & 0,09 \\
\hline Não & 11 & 40,74 & 27 & 0,09 \\
\hline \multicolumn{5}{|c|}{ Problemas com o cônjuge } \\
\hline Sim & 6 & 100 & 6 & 0,005 \\
\hline Não & 8 & 33,33 & 24 & 0,005 \\
\hline
\end{tabular}

UBS: Unidades Básicas de Saúde; PSF: Programa Saúde da Família

\section{Discussão}

As mulheres deste estudo apresentam um bom nível educacional (apenas uma é analfabeta), encontram-se ainda em fase produtiva e, mesmo que não trabalhem formalmente, muitas possuem algum tipo de ocupação.

Observou-se um percentual significativo dos sujeitos deste estudo $(56,6 \%)$ com sintomas indicativos de depressão, embora a amostra estudada seja pequena.

Analisando-se as respostas das mulheres às 21 questões do inventário de Beck, destacam-se a ocorrência
Tabela 4. Resultados de regressão logística das variáveis psicossociais relacionadas com depressão em mulheres no climatério

\begin{tabular}{lccc}
\hline \multicolumn{1}{c}{ Variáveis } & Razões de risco & $\mathbf{\pm}$ & $\boldsymbol{p}$ \\
\hline Hábito de beber & 16,88 & 30 & 0,112 \\
Ter companheiro & 7,96 & 9,87 & 0,095 \\
$\begin{array}{l}\text { Grupo etário } \\
\text { (50 anos ou mais) }\end{array}$ & $0,14^{*}$ & 0,2 & 0,156 \\
$\begin{array}{l}\text { Grupos biológicos } \\
\text { (B e C) }\end{array}$ & 5,65 & 5,23 & 0,062 \\
\hline
\end{tabular}

*Esse valor representa um fator de proteção. Se as categorias fossem invertidas, representaria um fator de risco $=1 / 0,144=6,925$.

e a composição dos sintomas característicos de depressão. Os sintomas mais sérios e que caracterizam um estado mais grave de depressão estiveram presentes sempre em uma das entrevistadas.

O sentimento de tristeza foi assinalado por 14 mulheres e somente uma sente-se tão triste que não consegue suportar; $73,3 \%$ delas têm esperanças no futuro e apenas $20 \%$ disseram que se sentiam desanimadas quanto ao futuro; 23 não se sentiam fracassadas e duas disseram que se sentiam um completo fracasso.

Cerca de $50 \%$ das mulheres sentiam prazer ao realizar as atividades cotidianas e $33,3 \%$ não o sentiam ao fazer as coisas como antes. Quatro delas sentem-se insatisfeitas e aborrecidas com tudo.

Sentimentos de culpa estavam sendo experimentados por quatro mulheres durante grande parte do tempo, embora 21 não se sentissem especialmente culpadas; outras quatro pacientes relataram que se sentem sempre culpadas. A possibilidade de ser punida foi referida por nove das entrevistadas, porém quatro achavam que estavam sendo punidas. O sentimento de decepção consigo é compartilhado por seis mulheres e quatro estão aborrecidas consigo mesmas. Com relação à crítica, dez relataram que se criticam por suas fraquezas e erros e três disseram que se culpam por tudo de mau que acontece. Entretanto a maioria (20) refere estar de bem consigo mesma.

Somente uma mulher disse que tinha ideação suicida e uma relatou que chora o tempo todo, enquanto a maioria (19) não chora mais do que o habitual e três dizem ser incapazes de chorar mesmo que o queiram.

$\mathrm{O}$ fato de se irritar mais facilmente do que costumavam foi assinalado por $53,3 \%$ das mulheres, e somente uma se irrita o tempo todo, entretanto nove não se irritam mais do que costumavam se irritar.

Duas perderam todo o interesse pelas outras pessoas e $60 \%$ não o perderam. Das 30 entrevistadas, nove estão menos interessadas nas pessoas do que costumavam. A preocupação com a aparência foi relatada por $30 \%$ das entrevistadas, enquanto $60 \%$ não estavam preocupadas com mudanças na aparência e duas se consideram feias.

A maioria $(60 \%)$ relatou que não tinha problemas para tomar suas decisões; $16,7 \%$ adiam as tomadas de decisões; $20 \%$ têm dificuldades ao tomar decisões; e somente uma não consegue mais tomá-las. 
A disposição para as atividades não estava alterada na maior parte das mulheres $(60 \%)$, embora seis precisassem de um esforço extra. Outras cinco precisavam se esforçar muito para fazer alguma coisa e uma não conseguia mais fazer trabalho algum.

Problemas com sono também foram relatados por 16 entrevistadas (dez não dormem como costumavam dormir, quatro acordam mais cedo e têm dificuldades para voltar a dormir e duas acordam várias horas mais cedo e não conseguem voltar a dormir).

O cansaço também é um sintoma freqüente na depressão. Foi assinalado por 17 entrevistadas que se cansam com mais facilidade; duas se cansam ao fazer qualquer coisa; e somente uma está cansada demais para iniciar qualquer atividade.

A maior parte (21) apresenta-se com o apetite sem alteração. Das 30 entrevistadas, nove têm problemas; em cinco 0 apetite não está tão bom como de costume; e quatro estão com o apetite pior. Com relação à perda de peso, 73\% disseram que não perderam peso e, entre as que perderam, a maioria o estava tentando. Metade das mulheres disse que se preocupava com problemas físicos e duas estavam tão preocupadas com problemas físicos que não conseguiam pensar em outra coisa.

O interesse por sexo não sofreu mudanças em somente sete delas; 13 relataram que não se interessam mais como antes; três estão menos interessadas atualmente; e sete perderam completamente 0 interesse.

Entre os eventos marcantes pelos quais as mulheres deste estudo passaram nos últimos 12 meses, os mais freqüentes foram: doenças $(33 \%)$, óbitos $(23 \%)$, problemas econômicos $(23 \%)$ e problemas com o cônjuge (20\%).

Portanto, observando-se que $63,3 \%$ das entrevistadas moram com companheiro, os resultados deste estudo levantam uma dúvida, pois não condiz com alguns achados da literatura (Santos et al., 2003), que afirmam que o fato de ter um companheiro seria um fator de proteção das mulheres contra a depressão.

Por outro lado, os achados relativos a problemas com o cônjuge e sentimentos negativos com relação a ele são preditivos de depressão, o que corrobora os achados da literatura (Silva et al., 2003; Veras e Nardi, 2005).

$\mathrm{Na}$ Tabela 3 observa-se que as não-brancas, em sua totalidade, apresentaram disforia/depressão, embora seja um grupo pequeno nesta amostra. Existem relatos na literatura de que as mulheres negras americanas referem mais sintomas depressivos do que as brancas (Viana et al., 2001).

Entre as entrevistadas, 56,7\% apresentavam uma ou mais doenças, sendo hipertensão arterial (HA) e diabetes mellitus (DM) as mais freqüentes. Para alguns estudiosos, a presença de doenças crônicas é fator de risco para a depressão (Wojnar et al., 2003), enquanto outros não encontraram essa associação (Veras AB, Nardi AE, 2005).

$O$ hábito de beber entre os familiares (observado em três casos) pode provocar desestabilização das relações intrafamiliares, sendo fator que contribui para o retraimento da mulher, bem como sua baixa auto-estima, por se sentir impotente diante da situação.

A ocorrência de óbito nos últimos 12 meses também predispõe à depressão, pois pode estar associado à vivência do luto. Esse fato foi observado em cinco casos.

Em vários estudos o desemprego é um fator altamente preditivo de sintomas de depressão (Gorenstein e Andrade, 1998; Silva et al., 2003; Veras e Nardi, 2005; Woods e Mitchell, 1996). O desemprego na família é um fator que levaria, conseqüentemente, a problemas de auto-estima, tendo em vistas as dívidas e outras dificuldades decorrentes do problema econômico e estaria diretamente relacionado à presença de reações depressivas.

A significativa existência de sinais de depressão deveria estar sendo acompanhada e orientada, tendo-se em vista a utilização de recursos de saúde por essas mulheres, principalmente nas UBSs. Entretanto elas podem não estar sendo adequadamente avaliadas.

Entre as cinco mulheres que utilizam o PSF não se encontrou depressão, e somente uma apresentou disforia, o que, em comparação com as que não têm esse tipo de assistência, é bastante significativo. Esse resultado reforça a importância dessa nova proposta de atendimento mais integrado e próximo.

Os sintomas depressivos entre as mulheres nessa fase da vida são bastante estudados na literatura internacional, embora pouco o sejam em nosso meio, especialmente pela enfermagem (Oliveira e Lemgruba, 2001).

Nesta amostra observou-se que os sintomas sugestivos de depressão em quase metade dessas mulheres podem estar relacionados à fase da vida que elas estão atravessando (do ponto de vista das alterações biológicas desse período) e a eventos psicossociais. Essas informações são de real importância para que a enfermagem planeje ações educativas, preventivas e curativas para essa população.

\section{Conclusão}

Os resultados mostraram que os sintomas depressivos estavam aumentados em mulheres na faixa de 40 a 49 anos, não-brancas e que vivenciaram o hábito de beber, situações de óbito recentemente e/ou de desemprego na família.

A influência de fatores psicossociais como faixa etária, presença e problemas com companheiro, cor, hábito de beber, ocorrência de óbito, recursos do sistema de saúde (UBS e PSF), desemprego, bem como o grupo biológico (presença de sintomas da menopausa), foi observada e discutida nesta pesquisa.

Em vista dos achados, sugerem-se pesquisas com maior número de indivíduos, além de estudos que favoreçam o cuidado de enfermagem e a atenção especializada a esse grupo de mulheres no que se refere aos sintomas de transtorno de humor na fase do climatério. 


\section{Referências}

Appolinário JC. A depressão na menopausa: uma entidade específica? Informativo Psiquiátrico, 18(4): 107-14, 1999.

Beck AT, Ward CH, Mendelson M, Mock J, Erbaugh J. An inventory for measuring depression. Archives of General Psychiatry, 4: 53-63, 1961.

Biffi EFA. O fenômeno menopausa: uma perspectiva de compreensão. 1991. Dissertação (Mestrado em Enfermagem). Escola de Enfermagem de Ribeirão Preto da Universidade de São Paulo, Ribeirão Preto.

Carranza-Lira S, Valentino-Figuero ML. Estrogen therapy for depression in postmenopausal women. International J Gynec \& Obst, 65: 35-8, 1999.

Coleman PM. Depression during the female climacteric period. Journal of Advanced Nursing, 18: 1540-6, 1993.

Dennersttein L, Lehert P, Burger H, Dudley E. Mood and the menopausal transition. The Journal of Nervous and Mental Disease, 187(11): 685-91, 1999.

Dias RS, Ramos CC, Kerr-Correa F, Trinca LA., Cerqueira ATAR, Dalben I, et al. Adaptação para o português do questionário de auto-avaliação de percepção de saúde física e mental da mulher de meia-idade: Questionário da Saúde da Mulher. Revista de Psiquiatria Clínica, 29(4): 181-9, 2002.

Freeman EW, Sammel MD, Liv L, Gracia CR, Nelson DB, Hollander L. Hormones and menopausal status as predictors of depression in women in transition to menopause. Archives General Psychiatry, 61: 62-70, 2004.

Genazzani AR, Monteleone P, Gambacciani M. Hormonal influence on the central nervous system. Maturitas, 43(supl 1): 11-7, 2002.

Gorenstein C, Andrade L. Inventário de depressão de Beck: propriedades psicométricas da versão em português. Revista de Psiquiatria Clínica, 25(5): 245-50, 1998.

Halbe HW. Tratado de ginecologia. 3 ed. São Paulo: Rocca; 2000.

lannetta O, Quintana SM, Ducati GB, Resener EV, Bregieiro LOR. Alterações neuroendócrinas no climatério. GO Atual,1(2): 25-33, 1997.
Lamberts SWJ, Van Den Beld AW, Van Der Lely A. The endocrinology of aging. Science, 278: 419-24, 1997.

Maartens LWF, Knottnerusb JA, Pop VJ. Menopausal transition and increased depressive syntomatology: a community based prospective study. Maturitas, 42: 195-200, 2002.

Miranda GCV, Figueira PG. Alterações psíquicas durante o climatério. Informativo Psiquiátrico, 18(4): 126-8, 1999.

Nicol-Smith L. Causality, menopause and depression: a critical review of the literature. British Medical Journal, 313: 1229-32, 1996.

Oliveira HC, Lemgruba I (coordenadores). Tratado de Ginecologia da FEBRASGO. Rio de Janeiro: Revinter Ltda.; 2001.

Perez IR, Pinar IM, Hernandez-Aguado I. Associated factors to psychiatric morbidity in postmenopausal phases. Maturitas, 24 : 107-15, 1997.

Richardson TA, Robinson RD. Menopausal and depression: a review of psychologie function and sex steroid neurobiology during the menopause. Primare Care, 7(6): 215-23, 2000.

Santos TM, Almeida AO, Martins HO, Moreno V. Aplicação de instrumento de avaliação do grau de depressão em universitários do interior paulista durante a graduação em enfermagem. Acta Scientiarum Acalth Sciences, 25(2): 171-6, 2003.

Silva MCF, Furegato ARF, Costa Junior ML. Depressão: pontos de vista e conhecimento de enfermeiros da rede básica de saúde. Revista Latinoamericana de Enfermagem, 11(1): 7-13, 2003.

Veras AB, Nardi AE. Hormônios sexuais femininos e transtornos do humor. Jornal Brasileiro de Psiquiatria, 54(1): 57-68, 2005.

Viana LC, Martins MMF, Geber S. Ginecologia. São Paulo: Medsi; 2001.

Wojnar M, Drózdz W, Araszkiewicz A, Szymanski W, Nawacka-Pawlaczyk D, Urbánski R, Hegedus AM. Assessment and prevalence of depression in women $45-55$ years of age visiting gynecological clinics in Poland. Archives of Women's Mental Health, 6: 193-201, 2003.

Woods NF, Mitchell ES. Patterns of depressed in midlife women: observations from the Seattle midlife women's health study. Research in Nursing \& Health, 19: 111-23, 1996. 